\title{
Agresión relacional en preescolar: variables cognoscitivas y emocionales asociadas*
}

\author{
Relational Aggression in Preschool: cognitive \\ and emotional variables associated
}

Recibido: julio 12 de 2012 | Revisado: mayo 14 de 2013 | Aceptado: julio 21 de 2013

\author{
CArlos Gómez-Garibello ** \\ McGill University, Montréla, Canadá \\ ENRIQUe CHAUX **** \\ Universidad de los Andes, Bogotá, Colombia
}

doi:10.11144/Javeriana.UPSY13-2.arpv

Para citar este artículo: Gómez-Garibello, C., \& Chaux, E. (2014). Agresión relacional en preescolar: variables cognoscitivas y emocionales asociadas. Universitas Psychologica, 13(2), 565-574. doi:10.11144/Javeriana.UPSY13-2.arpv

\footnotetext{
* Artículo de investigación

*** carlos.gomez-garibello@mail.mcgill.ca

**** echaux@uniandes.edu.co
}

\section{RESUMEN}

Cuando una persona usa la agresión relacional busca dañar las relaciones de otros a través de manipulaciones y exclusión de grupo. Existe muy poca investigación sobre este tipo de agresión en el preescolar. De igual forma, tampoco se han establecido con claridad qué variables psicológicas se asocian con esta. La presente investigación se centró en la agresión relacional y la agresión física en una muestra de 77 niños y niñas de preescolar. Se midieron variables cognoscitivas (creencias favorables hacia la agresión y teoría de la mente) y variables emocionales (control de la ira) en los niños. No se encontraron diferencias significativas entre niños y niñas en los niveles de agresión física o de agresión relacional. Sin embargo, cuando se incluyó agresión física como covariada, se encontró más agresión relacional en niñas que en niños y cuando agresión relacional fue usada como covariada, se encontró más agresión física en niños que en niñas. El control de la ira apareció como un importante factor en la predicción de la agresión relacional. Palabras clave

agresión; educación; escuela; desarrollo

\section{A B S T R A C T}

When somebody uses relational aggression seeks damage relations of others through manipulation and exclusion group. There is little research on this aggression type in preschool. Similarly, not been established clearly what psychological variables are associated with this. This research focused both relational and physical aggressions in a sample of 77 preschool children. Were measured cognitive variables (beliefs favorable toward aggression and theory of mind) and emotional variables (anger) in children. Do not significant differences between boys and girls were found in levels physical aggression or relational aggression. However, when included physical aggression as a covariate, more relational aggression was found in girls than children, and when relational aggression was used as a covariate, was found more physical aggression in boys than in girls. The anger came as an important factor in predicting relational aggression.

Keywords

agression; education; preschool; development 
La evidencia existente sugiere que los niños en edad preescolar pueden presentar comportamientos que se catalogarían como agresión relacional, es decir, comportamientos que buscan hacerle daño a las relaciones de otros (Bonica, Arnold, Fisher, Zeljo \& Yershova, 2003; Crick, Casas \& Ku, 1999; Crick, Casas \& Mosher, 1997; Crick et al., 2006). Una amplia cantidad de investigaciones, usando múltiples metodologías, ha descrito esta forma de agresión (Archer \& Coyne, 2005; Card, Stucky, Sawalani \& Little, 2008; Crick et al., 2006; Ostrov \& Keating, 2004). Sin embargo, hay escasa evidencia en relación con los factores cognoscitivos y emocionales asociados con la agresión relacional en niños de edad preescolar. La investigación en el campo de agresión relacional sugiere que factores cognoscitivos como el procesamiento de información social (Crick \& Dodge, 1994; Crick \& Werner, 1998; Crick, Grotpeter \& Bigbee, 2002), las creencias (Slaby \& Guerra, 1988; Zelli, Dodge, Lochman \& Laird, 1999) y la teoría de la mente (Renouf et al., 2010; Sutton, Smith \& Swettenham, 1999) influyen en la agresión de los niños. Por otra parte, algunos autores han sugerido que existen asociaciones entre procesos emocionales como la empatía y el control de la ira y la agresión en general (Arsenio \& Lemerise, 2001; Berkowitz, 1990; Chaux, 2012). Sin embargo, hay escasa evidencia en población preescolar sobre la relación entre la agresión relacional y variables como creencias favorables hacia la agresión, teoría de la mente, empatía y control de emociones.

El propósito de este estudio fue conocer cuáles factores cognoscitivos y emocionales se relacionan con la agresión relacional en los niños entre 5 y 7 años. Las variables cognoscitivas que se utilizaron fueron teoría de la mente y creencias positivas hacia la agresión. Como variables emocionales se usaron empatía y control de la ira.

\section{Agresión física y agresión relacional}

La agresión se puede definir de forma general como una acción que tiene como objeto hacerle daño a otra persona (Parke \& Slaby, 1983). Crick y Grotpeter (1995) clasifican la agresión en física, cuando la intención es hacer daño físico, verbal cuando se busca herir a través de palabras y relacional cuando se pretende dañar las relaciones que tiene la persona o su estatus dentro de un grupo. Como agresión física se pueden incluir comportamientos como golpear, patear, empujar, lanzar objetos o atentar de alguna forma contra la integridad física o los bienes de una persona (Crick \& Grotpeter, 1995). Björkqvist, Österman y Kaukiainen (1992) encontraron que la agresión física es más frecuente entre los niños varones, independiente de la edad (ver también, Card et al., 2008). Las mujeres, en cambio, parecen recurrir a otras formas de agresión, especialmente la relacional (Archer \& Coyne, 2005; Card et al., 2008; Crick \& Grotpeter, 1995). Entre los comportamientos que se pueden catalogar como agresiones relacionales se enumeran chismes, manipulación de las relaciones y exclusión de los grupos (Crick \& Grotpeter, 1995; Crick, Casas \& Mosher, 1997).

El estudio de la agresión relacional se enfrenta a varias dificultades. Por un lado, existen diversos términos tales como "agresión indirecta" y "agresión social" con significado similar, pero con definiciones operativas distintas (Archer \& Coyne, 2005; Gyles \& Heyman, 2005). Por otro lado, existe la creencia errónea de que las mujeres son las únicas que usan la agresión relacional (Gyles \& Heyman, 2005; Ostrov \& Keating, 2004). A pesar de que algunos estudios han mostrado que la agresión relacional es más frecuente entre niñas que entre niños (Crick, Casas \& Mosher, 1997; Crick \& Grotpeter, 1995), otros estudios no han encontrado diferencias (Card et al., 2008; Salmivalli \& Kaukiainen, 2004).

En segundo lugar, Björkqvist et al. (1992) sostienen que la agresión relacional o indirecta es el producto de un proceso evolutivo en la agresión que se inicia en la agresión física. A medida que los niños y las niñas avanzan en su maduración biológica, psicológica y social, tienen nuevas herramientas para interpretar la realidad, para interactuar y para agredir (Björkqvist et al., 1992).

Finalmente, la tercera dificultad es que son todavía pocos los estudios sobre la agresión relacional en edad preescolar (Bonica et al., 2003; Crick, Casas $\&$ Mosher, 1997; Crick et al., 2006). 


\section{Variables asociadas con la agresión relacional}

Arsenio y Lemerise (2001) señalan dos grupos de variables importantes a la hora de entender los comportamientos agresivos: variables referidas a procesos cognoscitivos, como por ejemplo la forma como se razona frente a la situación social y variables sobre procesos emocionales.

En cuanto a los factores cognoscitivos, Slaby y Guerra (1988) encontraron que los adolescentes agresivos y con rasgos antisociales definen los problemas sociales de una forma más hostil que los adolescentes no agresivos. En particular, justifican sus acciones agresivas y se proponen metas con sesgo hostil (Slaby \& Guerra, 1988). Por otra parte, varios estudios han mostrado que las personas que tienen creencias favorables hacia la agresión tienen mayor probabilidad de utilizar la agresión como un medio para la solución de problemas y de conflictos sociales (Chaux, 2012; Guerra, Huesmann \& Spindler, 2003; Musher-Eizenman et al., 2004; Zelli et al., 1999).

Arsenio y Lemerise (2001) afirman que otra variable cognoscitiva asociada a la agresión es la teoría de la mente, que se basa en la presunción de que las personas tienen mente y de que esta estructura una red de estados mentales conformada por pensamientos, ideas e imágenes que subyacen a todos los comportamientos, creencias, deseos y sentimientos (Butterworth, Harris, Leslie \& Wellman, 1991). En el caso particular de la agresión relacional, Renouf et al. (2009) encontraron que altos niveles de teoría de la mente están relacionados con altos puntajes de agresión relacional pero solamente en el caso de niños con niveles promedio o bajos de comportamientos prosociales. Este hallazgo es consistente con la complementariedad entre factores cognoscitivos y emocionales a la hora de estudiar la agresión relacional propuesta por algunos autores (Arsenio \& Lemerise, 2001; Renouf et al., 2009; Sutton et al., 1999).

En relación con variables emocionales, Eisenberg, Spinrad y Sadovsky (2006) definen la empatía como "una respuesta afectiva que se produce de la aprehensión o comprensión de un estado o condición emocional de otro y que es similar a lo que la otra persona está sintiendo o a lo que se esperaría que sintiera en una situación dada" (p. 518). Björkqvist, Österman y Kaukiainen (2000) señalan que el desarrollo de la empatía inhibe o al menos mitiga la ocurrencia de comportamientos violentos, en la medida en que el hecho de reconocer los sentimientos de las víctimas hace que se reduzca la disposición a generarles daño a otros. Estos autores, también encontraron que las personas con altos índices de empatía tenían mayor probabilidad de encontrar formas pacíficas y constructivas de resolver los conflictos (Björkqvist et al., 1999).

Otra variable emocional relevante en el estudio de la agresión es el control emocional. Blair, Benham, Kochanoff y Whipple (2004) definen control emocional como un proceso externo y también interno mediante el cual se monitorean, evalúan y modifican las reacciones emocionales para ajustarlas a las metas. El proceso de control emocional sigue un camino que va de la regulación externa (de los cuidadores o personas alrededor del niño) a interna. Uno de los caminos que, según Holodynski y Friedlmeier (2006), sigue el control emocional es aprender a aplazar la satisfacción de las necesidades y a coordinar estas dentro del contexto social. Después de este aplazamiento, los niños deben aprender a darle prioridad a sus necesidades dependiendo de las normas del ambiente. Una vez se dan estas condiciones, señalan los investigadores, aparecen nuevas emociones como el orgullo, la pena y la culpa; de esta forma los niños aprenden a orientar sus respuestas hacia reacciones positivas de los adultos y a evitar negativas. De esta forma los niños van desarrollando una habilidad de autoevaluación que en principio parte de la evaluación de las acciones que hacen los adultos. Holodynski y Friedlmeier afirman que hacia el final del preescolar los niños pueden evaluar "su propio desempeño independientemente de juicios externos, y recurriendo a sus propios juicios" (p. 152). En opinión de Blair et al. (2004) es importante estudiar el control emocional en el preescolar, dado que los niveles bajos se asocian con problemas de comportamiento como agresión. A su vez, estos comportamientos 
son señales de alerta de conductas antisociales en la adolescencia y en la adultez (Blair et al., 2004).

En resumen, se ha encontrado que los niños en edad preescolar pueden presentar tanto comportamientos agresivos físicos como relacionales. Sin embargo, aun cuando existe evidencia empírica que resalta la importancia de variables cognoscitivas y emocionales a la hora de estudiar la agresión en niños en edad escolar y adolescentes, existe escasa evidencia en el estudio de estos tipos de agresión en prescolares. El presente estudio responde a este vacío, buscando: 1 . Determinar si para una muestra de niños colombianos en edad preescolar también se encuentran diferencias entre los sexos o entre los cursos en los niveles de agresión relacional y física y 2. Establecer qué factores cognoscitivos y emocionales están asociados con la agresión relacional y física en preescolar.

\section{Método}

\section{Participantes}

En total participaron 77 niños ( $55.8 \%$ hombres) con un promedio de edad de 5.58 años $(D E=0.62)$; el $61 \%$ cursaban kínder. La media de edad para los niños de kínder fue 5.23 años y para transición, 6.14 años. Se solicitó consentimiento informado a los padres. Los niños que participaron pertenecen a un colegio privado, mixto, ubicado en Bogotá, Colombia, de nivel socioeconómico medio-alto y alto. Solamente participaron los niños que fueron autorizados por sus padres por medio de un consentimiento informado.

\section{Instrumentos y procedimiento}

Tanto niños como profesores reportaron información sobre las variables del estudio. Los niños evaluaron creencias favorables a la agresión, teoría de la mente y empatía, e hicieron nominación de pares sobre agresión relacional y física. Por su parte, los profesores evaluaron el control emocional. Los niños participaron en entrevistas individuales. Para evitar los problemas que puedan surgir por el nivel de habilidad y destreza verbal de los niños, el entrevistador (primer autor) leyó las instrucciones, preguntas o historias y registró las respuestas por escrito.

\section{Creencias sobre la agresión}

Las creencias que legitiman la agresión fueron evaluadas con nueve preguntas adaptadas a preescolar de un instrumento desarrollado para edad escolar por Chaux, Arboleda y Rincón (en revisión) ( $\alpha=$ 0.602). El proceso de adaptación incluyó un pilotaje para identificar si los niños entendían las afirmaciones y una evaluación por parte de profesoras de preescolar. Por ejemplo, las creencias que se midieron incluyeron "Ver peleas entre compañeros es divertido" o "Si se portan mal con uno, uno tiene que responder de la misma manera".

\section{Teoría de la mente}

Para evaluar teoría de la mente se utilizó un instrumento creado y validado por Chaparro y Fandiño (2006). El instrumento consta de dos historias, seguidas por dos preguntas sobre teoría de la mente $(\alpha=0.745)$. La primera historia era la misma para los niños pues los protagonistas eran un niño y una niña. La segunda historia tenía dos versiones, una para cada sexo, esto se hizo porque el protagonista de la historia es un individuo, que en el caso de participantes hombres era un niño y en el de las mujeres, una niña. Las historias se apoyaron en dibujos para ayudar a la comprensión de los participantes.

\section{Empatía}

Empatía fue medida con dos preguntas adicionales para cada una de las dos historias del mismo instrumento usado para medir teoría de la mente diseñado y validado por Chaparro y Fandiño (2006) $(\alpha=0.634)$. Las respuestas se clasificaron en 1 (evidencia de empatía) o 0 (no evidencia de empatía o no respondió la pregunta).

\section{Control de la ira}

Para medir control emocional se usó la escala de Rydell, Berlin y Bohlin (2003) que mide emocio- 
nalidad y control emocional y que fue validada por estos mismos autores con padres de niños y niñas entre 5 y 8 años $(\alpha=0.806)$. Entre las emociones negativas incluyeron ira, temor y tristeza. Para esta investigación se eligió como emoción negativa la ira. Seis profesoras evaluaron esta variable para cada uno de los niños participantes. Un ítem que se utilizó fue "Cuando el niño se pone furioso, es fácil para otros, incluyendo a los profesores, calmarlo?" Las opciones de respuesta van desde no aplica para nada (1) hasta aplica muy bien para este niño (5).

\section{Agresión física y relacional}

Para agresión física y relacional se utilizó el instrumento de Crick et al. (1997) Preschool Behavior Scale Peers (PBSP). Este instrumento consta de 12 comportamientos que se clasifican en tres grupos: agresión física, agresión relacional y comportamientos prosociales (cada grupo tiene cuatro comportamientos). Los niños hicieron nominación de pares utilizando fotografías para clasificar a sus compañeros en las dimensiones de agresión relacional, agresión física y comportamientos prosociales. Se calculó el número de nominaciones que cada niño había recibido y se dividió por el número de niños participantes en cada curso. Se le pidió a cada niño nominar hasta tres compañeros.

\section{Análisis de datos}

En primer lugar, se utilizó chi cuadrado $\left(\chi^{2}\right)$ para determinar si existían diferencias significativas por sexo en la frecuencia con la que respondieron estar de acuerdo con creencias favorables a la agresión. Luego, un análisis de covarianza (ANCOVA) 2 (sexo) X 2 (curso) se usó para establecer si las diferencias por sexo y curso eran significativas en los puntajes de agresión relacional y comportamientos prosociales después de controlar el efecto de agresión física y viceversa. Con el objetivo de examinar el grado de asociación entre las variables cognoscitivas y emocionales y los estilos de agresión, se calcularon correlaciones bivariadas. Finalmente, para encontrar las variables cognoscitivas y emocionales que mejor predecían los estilos de agresión se calcularon dos modelos de regresión (uno por cada tipo de agresión).

TABLA 1

Frecuencias de las creencias favorables hacia la agresión por sexo

\begin{tabular}{|c|c|c|c|c|c|c|}
\hline \multirow[t]{2}{*}{ Creencia } & \multicolumn{2}{|c|}{$\begin{array}{l}\text { SÍ } \\
\text { Frecuencia }\end{array}$} & \multicolumn{2}{|c|}{$\begin{array}{l}\mathrm{NO} \\
\text { Frecuencia }\end{array}$} & \multirow[b]{2}{*}{$\chi^{2}$} & \multirow[b]{2}{*}{$p$} \\
\hline & Mujeres & Hombres & Mujeres & Hombres & & \\
\hline Si alguien le pega a uno, uno tiene que pegarle también. & 2 & 3 & 32 & 40 & 0.37 & 0.85 \\
\hline $\begin{array}{l}\text { Si alguien le pone apodos o le dice malas palabras, uno } \\
\text { tiene que responderles igual. }\end{array}$ & 6 & 2 & 28 & 41 & 3.44 & 0.06 \\
\hline $\begin{array}{l}\text { Si alguien lo saca a uno de los grupos y no lo deja jugar, uno } \\
\text { también tiene que sacarlo de los grupos y no dejarlo jugar. }\end{array}$ & 5 & 7 & 28 & 36 & 0.02 & 0.89 \\
\hline $\begin{array}{l}\text { Está bien tratar mal a otro(a) si esa persona lo agredió a } \\
\text { uno primero. }\end{array}$ & 8 & 3 & 25 & 40 & 4.49 & $0.03 *$ \\
\hline Está bien ofender a alguien si esa persona no me cae bien. & 3 & 2 & 31 & 40 & 0.5 & 0.48 \\
\hline $\begin{array}{l}\text { Es importante que la gente sepa que uno es fuerte y que } \\
\text { con uno no se pueden meter. }\end{array}$ & 17 & 23 & 15 & 20 & 0 & 0.98 \\
\hline Ver peleas entre compañeros es divertido. & 3 & 4 & 31 & 39 & 0.01 & 0.94 \\
\hline $\begin{array}{l}\text { Si se portan mal con uno, uno tiene que responder de la } \\
\text { misma manera. }\end{array}$ & 4 & 5 & 30 & 38 & 0 & 0.99 \\
\hline Si uno trata mal a la gente, uno después se siente mal. & 10 & 12 & 24 & 31 & 0.02 & 0.89 \\
\hline
\end{tabular}

$* p<0.05$.

Fuente: elaboración propia 


\section{Resultados}

\section{Diferencias en variables cognoscitivas y emocionales por sexo o curso}

Los análisis estadísticos sugieren que los niños y las niñas difieren en solamente una de las creencias favorables hacia la agresión: "está bien tratar mal a otro si esta persona lo trató mal a uno primero". Los resultados indican (Tabla 1) que los niñas apoyan más esta creencia en comparación con las niños $\left(\chi^{2}(1)=4.5 ; p<0.05\right)$.

En relación con el curso, se encontró que los niños más grandes (Transición) consideran con más frecuencia que los más pequeños (Tabla 2) que después de tratar a alguien mal uno se siente mal $\left(\chi^{2}(1)=11.56 ; p<0.05\right)$.

Por otra parte, no se encontraron diferencias estadísticamente significativas por género en teoría de la mente, empatía, creencias y control emocional. En cambio, cuando se indagó por diferencias entre los cursos (Kínder y Transición), se encontró que los niños de Kínder obtuvieron puntajes más bajos en teoría de la mente $(M=2.31 ; D E=1.4)$ en comparación a los niños de transición $(M=3 ; D E$ $=0.83 ; t(73)=-2.13 ; p=0.04)$. Sorpresivamente, los niveles de empatía de los niños de Kínder ( $M$ $=3.75 ; \mathrm{DE}=0.72$ ) fueron significativamente más altos que los de transición $(M=3.42 ; D E=0.72$; $t(68)=2.68 ; p=0.01)$.

\section{Diferencias en agresiones física y relacional por sexo y cursos}

Se calcularon diferencias por sexo y entre cursos en dos de las variables dependientes (agresión relacional y comportamientos prosociales). No se encontraron diferencias significativas entre niños y niñas ni en agresión física $(t(75)=1.17, p=0.54)$ ni en agresión relacional $(t(75)=-0.62, p=0.54)$. Debido a que existe una alta correlación entre la agresión relacional y agresión física (ver Tabla 3 con las correlaciones entre las variables), se calculó un análisis de covarianza, utilizando como variable dependiente agresión relacional y como covariada agresión física.

Los resultados sugieren que los puntajes de los niños en agresión relacional $(\mathrm{M}=0.12 ; \mathrm{DE}=0.12)$ eran significativamente más bajos que los de las niñas $(M=0.17 ; D E=0.16)$, cuando se controla el efecto de agresión física: $F(1,72)=4.54, p=0.03$. Cuando se utilizó agresión relacional como cova-

TABLA 2

Frecuencias de las creencias favorables hacia la agresión por curso

\begin{tabular}{|c|c|c|c|c|c|c|}
\hline \multirow{2}{*}{ Creencia } & \multicolumn{2}{|r|}{ SÍ } & \multicolumn{2}{|c|}{$\mathrm{NO}$} & \multirow[b]{2}{*}{$\chi^{2}$} & \multirow[b]{2}{*}{$p$} \\
\hline & Kínder & Transición & Kínder & Transición & & \\
\hline Si alguien le pega a uno, uno tiene que pegarle también. & 2 & 3 & 45 & 27 & 0.1 & 0.32 \\
\hline $\begin{array}{l}\text { Si alguien le pone apodos o le dice malas palabras, uno tiene } \\
\text { que responderles igual. }\end{array}$ & 7 & 1 & 40 & 29 & 2.63 & 0.11 \\
\hline $\begin{array}{l}\text { Si alguien lo saca a uno de los grupos y no lo deja jugar, uno } \\
\text { también tiene que sacarlo de los grupos y no dejarlo jugar. }\end{array}$ & 6 & 6 & 40 & 24 & 0.66 & 0.42 \\
\hline $\begin{array}{l}\text { Está bien tratar mal a otro(a) si esa persona lo agredió a uno } \\
\text { primero. }\end{array}$ & 5 & 6 & 41 & 24 & 1.22 & 0.27 \\
\hline Está bien ofender a alguien si esa persona no me cae bien. & 3 & 2 & 43 & 28 & 0 & 0.98 \\
\hline $\begin{array}{l}\text { Es importante que la gente sepa que uno es fuerte y que con } \\
\text { uno no se pueden meter. }\end{array}$ & 25 & 15 & 21 & 14 & 0.05 & 0.82 \\
\hline Ver peleas entre compañeros es divertido. & 6 & 1 & 41 & 29 & 1.97 & 0.16 \\
\hline $\begin{array}{l}\text { Si se portan mal con uno, uno tiene que responder de la } \\
\text { misma manera. }\end{array}$ & 6 & 3 & 41 & 27 & 0.14 & 0.71 \\
\hline Si uno trata mal a la gente, uno después se siente mal. & 20 & 2 & 27 & 28 & 11.56 & 0* \\
\hline
\end{tabular}

$* p<0.01$.

Fuente: elaboración propia 
TABLA 3

Correlación entre las variables medidas

\begin{tabular}{lcccccc}
\hline & Creencias & Empatía & $\begin{array}{c}\text { Control } \\
\text { emocional }\end{array}$ & $\begin{array}{c}\text { Agresión } \\
\text { relacional }\end{array}$ & $\begin{array}{c}\text { Agresión } \\
\text { física }\end{array}$ & $\begin{array}{c}\text { Comp. } \\
\text { prosociales }\end{array}$ \\
\hline Teoría de la mente & -0.204 & 0.103 & -0.023 & -0.055 & -0.083 & 0.006 \\
Creencias & & -0.22 & -0.126 & 0.106 & 0.165 & -0.15 \\
Empatía & & -0.12 & 0.162 & 0.161 & 0.046 \\
Control Emocional & & & & $-0.322^{* *}$ & $-0.422^{* *}$ & 0.158 \\
Agresión relacional & & & & & $0.723 * *$ & 0.175 \\
Agresión Física & & & & & 0.042 \\
\hline
\end{tabular}

$* * p<0.01$.

Fuente: elaboración propia

riada, los niños $(M=0.16 ; D E=0.15)$ mostraron puntajes significativamente más altos que las niñas $(M=0.12 ; D E=0.14 ; F(1,74)=5.80, p=0.02)$. No se encontraron diferencias significativas entre cursos en agresión relacional después de controlar el efecto de la agresión física, ni en agresión física después de controlar agresión relacional.

\section{Variables cognoscitivas y emocionales predictoras de agresión relacional}

El primer modelo incluyó agresión relacional como variable dependiente y los cuatro predictores mencionados (creencias, control emocional, teoría de la mente y empatía). El modelo fue significativo, aunque control de la ira fue el único predictor significativo (Tabla 4). En cuanto a agresión física, el modelo también fue significativo y, de nuevo, control de la ira fue el único predictor significativo (Tabla 4).

\section{Discusión}

La literatura sobre agresión coincide en que se trata de fenómeno que se presentan desde los primeros años de vida (Tremblay et al., 2004). De acuerdo con los datos obtenidos en este estudio, los participantes vivencian agresión física y agresión relacional en edad preescolar. En efecto, se encontró una alta correlación entre la agresión física y la relacional, esto es, los niños que utilizan la agresión física, también en un alto grado utilizan la agresión relacional en sus interacciones. En este sentido, vale la pena recordar los señalamientos de Björkqvist et al. (1992), quienes consideran que las dos formas de agresión parecen seguir un patrón en el que la agresión física es reemplazada paulativamente por la agresión relacional.

En cuanto a las variables cognoscitivas, se encontró que los niños diferían solamente en una

TABLA 4

Modelos de regresión de agresión relacional y física, respectivamente

\begin{tabular}{lcc}
\hline Variable & Agresión relacional & Agresión física \\
\hline Constante & $\beta$ & $\beta$ \\
Teoría de la mente & & -0.059 \\
Empatía & -0.092 & 0.147 \\
Control emocional & 0.174 & $-0.497^{* *}$ \\
Creencias favorables a la agresión & $-0.343^{* *}$ & 0.152 \\
$R^{2}$ & 0.121 & 0.32 \\
$F$ & 0.18 & $6.815^{*}$ \\
\hline
\end{tabular}

$* p<0.05, * * p<0.01$.

Fuente: elaboración propia

\begin{tabular}{l|l|l|l|l|} 
& Universitas Psychologica & V. 13 & No. 2 & abril-junio |
\end{tabular} 
creencia favorable hacia la agresión ("está bien tratar mal a otro, si esta persona lo trato mal a uno primero"). Dado que las niñas mostraron un mayor nivel de aceptación de esta creencia, una posible explicación podría ser que entendieron mejor estas creencias que los niños. Sin embargo, se requiere más investigación para clarificar el papel de las creencias en la explicación de comportamientos agresivos en preescolar. Por otra parte, se encontró que los niños mayores están más de acuerdo que los niños de Kínder con la creencia "después de agredir a alguien, la gente se siente mal". Este resultado no es sorprendente en la medida en que otros estudios han mostrado que los niños van desarrollando empatía a medida que van madurando (Eisenberg et al., 2006).

Los análisis estadísticos realizados señalan que las creencias no son un predictor significativo de los comportamientos agresivos, al menos en los niños que participaron en la presente investigación. Este hecho, contradice algunos resultados de investigaciones realizadas en el campo de la agresión (Chaux et al., en revisión; Slaby \& Guerra, 1988; Zelli et al., 1999). Una posible explicación podría estar en que los niños probablemente confundieron las creencias con descripciones de lo que ellos ven que ocurre. En este sentido, por ejemplo ante la pregunta "icrees tú que si alguien le pone apodos o le dice malas palabras a uno, uno tiene que responderles igual?", algunos niños hayan respondido pensando en que se les estaba preguntando "ite ha pasado que cuando alguien le pone apodos o le dice malas palabras a uno, uno les responde igual?".

En cuanto a teoría de la mente, como era de esperarse los niños de Transición mostraron valores más altos que los más pequeños. Esto es consistente con las trayectorias de desarrollo que siguen las características cognoscitivas como la mencionada teoría (Keenan, 2003).

El estudio mostró que los niños de Transición tenían valores más bajos de empatía en comparación a los de Kínder. Este hallazgo es sorprendente, toda vez que los estudios previos en esta área señalan que los niños van desarrollando empatía a medida que pasa el tiempo y tienen la oportunidad de interactuar con más personas (Björkqvist et al.,
1992; Eisenberg, 2000). Además, este resultado no es consistente con el hecho de que los niños más grandes apoyan en mayor proporción la creencia de que después de tratar mal a alguien uno se siente mal. Se requiere más investigación en el punto específico de la relación entre empatía y creencias favorables hacia la agresión. Finalmente, no se encontraron diferencias significativas por cursos en el control emocional de la ira. Esto tal vez puede ser consecuencia de que la diferencia entre el desarrollo emocional de los niños de Kínder y Transición no es suficiente para dar cuenta de diferencias en el control de la ira.

Debido a la ya comentada correlación entre agresión física y relacional, se controló el efecto de una sobre la otra para analizar diferencias por sexo. De esta forma, se encontraron niveles más altos de agresión relacional entre niñas que entre niños, y niveles más altos de agresión física entre niños que entre niñas. Este resultado es consistente con buena parte de la literatura en el área de agresión relacional y física en general (Card et al., 2008; Crick \& Grotpeter, 1995; Salmivalli \& Kaukiainen, 2004) y en edad prescolar en particular (Crick et al., 1997; Crick et al., 2006; Ostrov \& Keating, 2004).

Los resultados de esta investigación revelan que el control de la ira es una variable importante para entender la agresión en los niños en preescolar. Se encontró que el control de la ira predijo tanto agresión física como relacional. En particular, niños y niñas con alto nivel de control de la ira reportado por sus profesoras tienden a tener bajos niveles de agresión física y relacional reportados por sus compañeros. Los resultados coinciden con lo señalado por Holodynski y Friedlmeier (2006) quienes afirman que el proceso de adquisición del control de emociones hace que los niños sean cada vez más conscientes del efecto que tiene la expresión de sus emociones en los demás y de la necesidad de ajustar esta expresión dentro del contexto social. La agresión se relaciona con el control de emociones negativas, principalmente con el control de la ira (Berkowitz, 1990). Los niños están aprendiendo a aplazar la satisfacción de sus necesidades y este aplazamiento incluye el manejo de la ira y de la frustración. 


\section{Limitaciones y aportes del estudio}

Una limitación de este estudio es el tamaño de la muestra y el tipo de muestreo utilizado (muestreo por conveniencia). La muestra es de un grupo urbano con relativamente alto nivel socioeconómico, lo que impide generalizar los resultados a otros grupos. Además, es preciso tener en cuenta posibles falencias de algunas escalas que fueron desarrolladas en estudios previos por otros investigadores y que se tomaron como antecedentes instrumentales para el presente estudio. Se necesita más investigación en el tema de cómo evaluar variables cognoscitivas relacionadas con la agresión en el contexto de preescolar.

El aporte de la presente investigación está en la verificación de las funciones de variables cognoscitivas y emocionales para la explicación de la agresión relacional en la niñez preescolar. Además, los hallazgos apoyan los reportados por Bonica et al. (2003), Crick, Casas y Mosher (1997), Crick et al. (2006) y Crick et al. (1997), entre otros autores, sobre la ocurrencia de comportamientos propios de la agresión relacional en prescolares. La información puede ser de especial utilidad para profesores por cuanto aportan evidencia de que las agresiones que ocurren en sus salones no se refieren exclusivamente a golpes y empujones, sino también a daños a las relaciones de otros, por ejemplo, a través de chismes o exclusión de grupos. Por otra parte, profesionales dedicados a diseñar programas de prevención de la agresión escolar, pueden encontrar particularmente interesante el rol del control de la ira en la aparición de las agresiones física y relacional en preescolares.

\section{Referencias}

Archer, J., \& Coyne, S. M. (2005). An integrated review of indirect, relational and social aggression. Personality and Social Psychology Review, 9((3), 212-230. doi:10.1207/s15327957pspr0903_2

Arsenio, W. F., \& Lemerise, E. A. (2001). Varieties of childhood bullying: Values, emotion processes, and social competence. Social Development, 10(1), 59-73. doi:10.1111/1467-9507.00148
Berkowitz, L. (1990). On the formation and regulation of anger and aggression: A cognitive-neoassociationistic analysis. American Psychologist, 45 (4), 494-503. doi:10.1037/0003-066X.45.4.494

Björkqvist, K., Lagerspetz, K. M. J., \& Kaukiainen, A. (1992). Do girls manipulate and boys fight? Developmental trends in regard to direct and indirect aggression. Aggressive Behavior, 18(2), 117-127.

Björkqvist, K., Österman, K., \& Kaukiainen, A. (2000). Social intelligence - empathy $=$ aggression? Aggression and Violent Behavior, 5(2), 191-200. doi:10.1016/ S1359-1789(98)00029-9

Blair, K., Denham, S., Kochanoff, A., \& Whipple, B. (2004). Playing it cool: Temperament, emotion regulation, and social behavior in preschoolers. Journal of School Psychology, 42(6), 419-443. doi:10.1016/j. jsp.2004.10.002

Butterworth, G., Harris, P, Leslie, A., \& Wellman, H. (1991). Perspectives on the Child's Theory of Mind. Oxford: Oxford University Press.

Card, N. A., Stucky, B. D., Sawalani, G. M., \& Little, T. D. (2008). Direct and indirect aggression during childhood and adolescence: A meta-analytic review of gender differences, intercorrelations, and relations to maladjustment. Child Development, 79(5), 1185-1229. doi:10.1111/j.1467-8624.2008.01184.x

Chaux, Arboleda \& Rincón (en revisión).

Chaparro, M. P., \& Fandiño, A. (2006). Validación de métodos de evaluación de competencias ciudadanas en niños de edad preescolar. Trabajo de Grado, Departamento de Psicología, Universidad de Los Andes, Bogotá, Colombia.

Crick, N. R., Casas, J. F., \& Ku, H.C. (1999). Relational and physical forms of peer victimization in preschool. Developmental Psychology, 35(2), 376-385. doi:10.1037/0012-1649.35.2.376

Crick, N. R., Casas, J. F., \& Mosher, M. (1997). Relational and overt aggression in preschool. Developmental Psychology, 33(4), 579-588. doi:10.1037/00121649.33.4.579

Crick, N. R., \& Dodge, K. A. (1994). A review and reformulation of social information-processing mechanisms in children's social adjustment. Psychological Bulletin, 115(1), 74-101. doi:10.1037/00332909.115.1.74 
Crick, N. R., Grotpeter, J. K., \& Bigbee, M. A. (2002). Relationally and physically aggressive children's intent attributiions and feelings of distress for relational and instrumental peer provocations. Child Development, 73(4), 1134-1142. doi:10.1111/14678624.00462

Crick, N. R., Ostrov, J. M., Burr, E. J., Cullerton-Sen, C., Jansen-Yeh, E., \& Ralston, P. (2006). A longitudinal study of relational and physical aggression in preschool. Journal of Applied Developmental Psychology, 27(3), 254-268. doi:10.1016/j.appdev.2006.02.006

Crick, N. R., \& Werner, N.E. (1998). Response decision processes in relational and overt aggression. Child Development, 69(6), 1630-1639. doi:10.1111/j.1467-8624.1998.tb06181.x

Eisenberg, N. (2000). Prosocial development in childhood and adolescence. En Encyclopedia of Psychology (Vol. 6, pp. 329-332). Washington: American Psychological Association Oxford University Press.

Eisenberg, N., Spinrad, T., \& Sadovsky, A. (2006). Empathy-related responding in children. En M. Killen \& J. G. Smetana (Eds.), Handbook of moral development (pp. 517-549). Mahwah, NJ: Lawrence Erbaum Associates Publishers.

Giles, J. W., \& Heyman, G. D. (2005). Young children's beliefs about the relationship between gender and aggressive behavior. Child Development, 76(1), 107 121. doi:10.1111/j.1467-8624.2005.00833.x

Guerra, N., Huesmann, R., \& Spindler, A. (2003). Community violence exposure, social cognition and aggression among urban elementary school children. Child Development, 74(5), 1561-1576. doi:10.1111/1467-8624.00623

Holodynski, M., \& Friedlmeier, W. (2006). Development of emotions and emotion regulation. New York: Springer.

Musher-Eizenman, D. R., Boxer, P., Danner, S., Dubow, E. F., Goldstein, S. E., \& Heretick, D. M. L. (2004). Social-cognitive mediators of the relation of environmental and emotion regulation factors to children's aggression. Aggressive Behavior, 30(5), 389-408. doi: 10.1002/ab.20078
Ostrov, J. M., \& Keating, C. F. (2004). Gender differences in preschool aggression during free play and structured interactions: an observational study. Social Development, 13(2), 255-277. doi:10.1111/j.14679507.2004.000266.x

Parke, R. D., \& Slaby, R. G. (1983). The development of aggression. En P. H. Mussen \& E. M. Hetherington (Eds.), Handbook of Child Psychology. Socialization, personality, and social development (Vol. 4, pp. 567-641). New York, USA: Wiley.

Renouf, A., Brendgen, M., Parent, S., Vitaro, F., Zelazo, P. D., Boivin, M., Dionne, G., et al. (2010). Relations between theory of mind and indirect and physical aggression in kindergarten: Evidence of the moderating role of prosocial behaviors. Social Development, 19(3), 535-555. doi:10.1111/j.14679507.2009.00552.x

Rydell, A., Berlin, L., \& Bohlin, G. (2003). Emotionality, emotion regulation, and adaptation among 5-to-8-old-year children. Emotion, 3(1), 30-47. doi:10.1037/1528-3542.3.1.30

Salmivalli, C., \& Kaukiainen, A. (2004). "Female aggression" revisited: Variable-and person-centered approaches to studying gender differences in different types of aggression. Aggressive Behavior, 30(2), 158-163. doi:10.1002/ab.20012

Sutton, J., Smith, P. K., \& Swettenham, J. (1999). Social cognition and bullying: Social inadequacy or skilled manipulation? British Journal of Developmental Psychology, 17(3), 435-450. doi:10.1348/026151099165384

Tremblay, R. E., Nagin, D. S., Seguin, J. R., Zoccolillo, M., Zelazo, P. D., Boivin, M., Perusse, D., et al. (2004). Physical aggression during early childhood: trajectories and predictors. Pediatrics, 114(1), 43-50. doi:10.1542/peds.114.1.e43

Zelli, A., Dodge, K. A., Lochman, J. E., \& Laird, R. D. (1999). The distinction between beliefs legitimizing aggression and deviant processing of social cues: Testing measurement validity and the hypothesis that biased processing mediates the effects of beliefs on aggression. Journal of Personality and Social Psychology, 77(1), 150-166. doi:10.1037/00223514.77.1.150 